\title{
MiR-506 Promotes Natural Killer Cell Cytotoxicity against Human Hepatocellular Carcinoma Cells by Targeting STAT3
}

\author{
Zhixiong $\mathrm{Su}^{1 *}$, Xinping $\mathrm{Ye}^{2 *}$, and Liming Shang ${ }^{2}$ \\ ${ }^{1}$ Department of General Surgery, Second Affiliated Hospital of Guangxi Medical University, Nanning; \\ ${ }^{2}$ Department of Hepatobiliary Surgery, First Affiliated Hospital of Guangxi Medical University, Nanning, China.
}

\begin{abstract}
Purpose: It is well documented that natural killer (NK) cytotoxicity against hepatocellular carcinoma (HCC) cells is impaired in HCC, which might account for the failure of anti-tumor immune response. miRNAs are considered as important regulators for the development and functions of NK cells. However, the entire role of miR-506 in NK cells remains far from being addressed. Materials and Methods: The expressions of miR-506 and signal transducer and activator of transcription 3 (STAT3) mRNA in primary NK cells from HCC patients and healthy controls were detected by quantitative real-time PCR. NK cell cytotoxicity was assessed by CFSE/7AAD cytotoxicity assay and lactate dehydrogenase assay. Luciferase reporter assay, RNA immunoprecipitation assay, and western blot were conducted to confirm the interaction between miR-506 and STAT3.

Results: miR-506 expression was downregulated and STAT3 mRNA was upregulated in primary NK cells from HCC patients. Primary NK cells from HCC patients showed remarkably reduced cytotoxicity against SMMC7721 or HepG2 cells. NK cell cytotoxicity was positively correlated with miR-506 expression and negatively correlated with STAT3 mRNA expression. Additionally, miR506 overexpression enhanced NK cell cytotoxicity against HCC cells, while miR-506 inhibitor showed the reverse effect. Moreover, miR-506 could suppress STAT3 expression by directly targeting 3'-untranslated regions of STAT3. A negative correlation between miR-506 and STAT3 mRNA expression in HCC patients was observed. Mechanistically, overexpressing STAT3 greatly reversed miR-506-mediated promotion of NK cell cytotoxicity against HCC cells.
\end{abstract}

Conclusion: miR-506 enhanced NK cell cytotoxicity against HCC cells by targeting STAT3, suggesting that modulating miR-506 expression maybe a promising approach for enhancing NK cell-based antitumor therapies.

Key Words: miR-506, NK cell cytotoxicity, STAT3, hepatocellular carcinoma

\section{INTRODUCTION}

Hepatocellular carcinoma (HCC) is currently known as one of the deadliest types of cancer as well as the second-leading cause

Received: June 8, 2018 Revised: August 22, 2018

Accepted: August 23, 2018

Corresponding author: Zhixiong Su, MD, Department of General Surgery, Second Affiliated Hospital of Guangxi Medical University, Nanning, Guangxi 530007, China. Tel: 86-0771-3277068, Fax: 86-0771-3277285, E-mail: y2521790543@163.com

*Zhixiong Su and Xinping Ye contributed equally to this work.

- The authors have no potential conflicts of interest to disclose.

(C) Copyright: Yonsei University College of Medicine 2019

This is an Open Access article distributed under the terms of the Creative Commons Attribution Non-Commercial License (https://creativecommons.org/licenses/ by-nc/4.0) which permits unrestricted non-commercial use, distribution, and reproduction in any medium, provided the original work is properly cited. of cancer-related mortality worldwide. ${ }^{1}$ As a major barrier to cancer progression, the immune system has the ability to eliminate tumor cells before they invade or progress during carcinogenesis. Natural killer (NK) cells, a major component of the innate immune response, play an important role in the immune function in liver and act as the first line of immune defense against viral infections and tumors. ${ }^{2}$ It is well documented that NK cells are abundant in the liver and essential for antihepatoma activity. ${ }^{3}$ NK cytotoxicity against HCC cells is impaired in HCC, which might account for the failure of anti-tumor immune response. ${ }^{4,5}$ Moreover, the number of liver NK cells is also dramatically diminished during HCC progression, and decreased NK cells in peripheral blood of patients with HCC is significantly correlated with poor prognosis of HCC. ${ }^{6}$ Therefore, enhancement of NK cells' cytotoxicity against tu- 
mors might be a promising immunotherapy approach.

microRNAs (miRNAs) are a class of small non-coding RNAs, which mediate the regulation of gene expression via recognizing the complementary sequences within 3 '-untranslated regions (3'-UTR) of target mRNAs, leading to mRNA degradation or translational inhibition. ${ }^{7}$ Over the past few years, increasing studies have shown that miRNAs are frequently dysregulated in cancers, consequently affecting diverse physiological and pathological processes such as proliferation, invasion, and immune escape. ${ }^{8}$ In the immune system, miRNAs play significant roles in regulating the development and function of B-cell subsets, regulatory T-cells, and myeloid lineage cells. ${ }^{9}$ Moreover, recent studies have shown direct evidence that miRNAs are considered important regulators for the development and functions of NK cells. ${ }^{10}$ miR-506, located on Xq27.3, was previously identified as a component of X chromosome-linked miRNA cluster in testes of primates. ${ }^{11}$ miR-506 has been reported to function either as oncogene or tumor suppressor in different types of malignancies. ${ }^{12}$ Importantly, previous studies reported that miR-506 was downregulated in HCC tissues compared to adjacent normal tissues, and acted as a tumor suppressor miRNA in HCC. However, the entire role of miR-506 in NK cells remains far from being addressed.

In our study, we demonstrated that miR-506 expression was positively correlated with NK cell cytotoxicity isolated from peripheral blood of HCC patients. Moreover, ectopic expression of miR-506 significantly enhanced NK cell cytotoxicity by targeting signal transducer and activator of transcription 3 (STAT3), a transcriptional factor that regulates all aspects of NK cell biology. Our study suggested that modulating miR-506 expression maybe a promising approach for enhancing NK cell-based antitumor therapies.

\section{MATERIALS AND METHODS}

\section{Subjects and isolation of NK cells}

Written informed consent was obtained from all participants, and our study was approved by Medical Ethics Committee of First Affiliated Hospital of Guangxi Medical University. A total of 15 HCC patients and 15 healthy donors, who were recruited at the Hepatobiliary Surgery Department of First Affiliated Hospital of Guangxi Medical University, were included in our study. Peripheral blood mononuclear cells (PBMCs) were isolated from HCC patients and healthy controls by density gradient centrifugation (Human lymphoprep, TBD, Tianjin, China). Primary NK cells were negatively selected from PBMCs using magnetic activated cell sorting human NK cell isolation kit (Sangon Biotech, Shanghai, China).

\section{NK cell culture and transfection}

HCC cell line SMMC7721, HepG2, human NK cell line NK-92, and 293T cells were purchased from American Type Culture
Collection (ATCC, Manassas, VA, USA). SMMC7721, HepG2, and 293T cells were maintained in DMEM with $10 \%$ heat-inactivated fetal bovine serum (FBS; Sigma-Aldrich, St. Louis, MO, USA) and $1 \%$ penicillin/streptomycin. NK-92 cells were maintained in $\alpha$-minimum essential medium (Invitrogen, Carlsbad, CA, USA) supplemented with $12.5 \%$ FBS (Sigma-Aldrich), $12.5 \%$ horse serum (Gibco, Grand Island, NY, USA), $100 \mathrm{U} / \mathrm{mL}$ recombinant human IL (rhIL)-2 (PeproTech, Rocky Hill, NJ, USA), and $0.1 \mathrm{mM} \beta$-mercaptoethanol. The isolated primary NK cells were cultured in complete RPMI medium (Gibco) supplemented with $10 \%$ heat-inactivated FBS (Sigma-Aldrich), $2 \mathrm{mM}$ L-glutamine, and $100 \mathrm{U} / \mathrm{mL}$ rhIL-2 (PeproTech). All cells were incubated at $37^{\circ} \mathrm{C}$ in an incubator of $5 \% \mathrm{CO}_{2}$.

miR-506 mimic (miR-506), miRNA negative control (miRNC), miR-506 inhibitor (anti-miR-506), inhibitor control (antimiR-NC), pcDNA-STAT3, pcDNA empty control (pcDNA), siRNA specially against STAT3 (si-STAT3), and siRNA control (siNC) were purchased from GenePharma (Shanghai, China). NK-92 and primary NK cells were seeded into 96-well plates and transfected with the abovementioned nucleotides or plasmids, using Lipofectamine 2000 (Invitrogen). Co-culturing or RNA extraction was performed 48 hours after transfection.

\section{Quantitative real-time PCR}

Total RNA, including miRNA, was isolated from the isolated primary NK cells or NK-92 cells using TRIzol reagent (Invitrogen). Complementary DNA was synthesized from $1 \mu \mathrm{g}$ of total RNA using PrimeScript RT reagent kit (TaKaRa, Dalian, China). miR-506 detection was performed using TaqMan miRNA assay system (Applied Biosystems, Foster City, CA, USA), while STAT3 mRNA expression was examined using SYBR Green Real-time PCR Master Mix (TaKaRa). Quantitative real-time (qRT) PCR reaction was conducted on GeneAmp PCR 9700 Thermo cycler (Applied Biosystems). Expressions of miR-506 and STAT3 mRNA were evaluated using $2^{-\Delta \Delta \mathrm{Ct}}$ method.

\section{Cytotoxicity assay}

NK-92 cell-mediated cytotoxicity against HCC cells was assessed via flow cytometry using CFSE/7AAD cytotoxicity assay. NK-92 cells transfected with or without miR-506, miR-NC, anti-miR-506, anti-miR-NC, miR-506 + pcDNA, miR-506 + STAT3, anti-miR-506 + si-NC, or anti-miR-506 + si-STAT3 were labeled with $5 \mu \mathrm{M}$ CFSE (Beyotime, Nanjing, China) for 10 $\min$ at $37^{\circ} \mathrm{C}$. Stained cells were washed twice with phosphatebuffered saline (PBS) and mixed with target SMMC7721 cells at various effector-to-target ratios (E:T): 10:1, 5:1, and 2.5:1, followed by $6 \mathrm{~h}$ of incubation at $37^{\circ} \mathrm{C}$. After washed twice with PBS, cell mixtures were and incubated with 7-AAD (KeyGEN BioTECH, Nanjing, China) for $15 \mathrm{~min}$ and detected using FACSCalibur system (BD Biosciences, San Jose, CA, USA).

Primary NK cell cytotoxicity was determined using a lactate dehydrogenase (LDH) assay according to the manufacturer's instructions. Briefly, SMMC7721 or HepG2 cells were seeded 
into 96-well plates at a density of $5 \times 10^{3}$ cells per well before coculturing. Primary NK cells transfected with or without miR506, miR-NC, anti-miR-506, anti-miR-NC, miR-506 + pcDNA, miR-506 + STAT3, anti-miR-506 + si-NC, or anti-miR-506 + siSTAT3 were added to each well and co-cultured with SMMC 7721 or HepG2 cells at an E:T ratio of 5:1. After $4 \mathrm{~h}$ of incubation, supernatant was collected and cytolytic activity of NK cells was examined using LDH Activity Assay kit (Jiancheng Bioengineering, Nanjing, China).

\section{Luciferase reporter assay}

Wild-type or mutated STAT3 3'UTR fragments containing the potential miR-506 binding sites were synthesized and cloned into pMIRNA-Reporter vector (Ambion, Austin, TX, USA) to produce pMIRNA-STAT3-WT (STAT3-WT) and pMIRNA-STAT3MUT (STAT3-MUT). 293T cells were seeded into 24-well plates and cotransfected with miR-506 or miR-NC and constructed luciferase plasmids using Lipofectamine 2000 (Invitrogen). After $48 \mathrm{~h}$ of transfection, firefly and Renilla luciferase activity was performed using Dual Luciferase Reporter Assay Kit (Promega, Madison, WI, USA).

\section{Western blot}

Cell protein was extracted from primary NK cells transfected with miR-506, anti-miR-506, or matched controls using RIPA lysis buffer (Sigma-Aldrich) for $10 \mathrm{~min}$ at $4^{\circ} \mathrm{C}$ and quantified using Bio-Rad protein assay system (Bio-Rad, Hercules, CA, USA). Equal amounts of cellular proteins were loaded on $10 \%$ sodium dodecyl sulfate-polyacrylamide (SDS-PAGE) gel electrophoresis and transferred to polyvinylidene difluoride membranes (PVDF, Millipore, Billerica, MA, USA). Membranes were blocked with $5 \%$ nonfat milk at room temperature for $2 \mathrm{~h}$, and then incubated with primary antibody against STAT3 (1:1000; Cell Signaling Technology, Beverly, MA, USA) and $\beta$-actin (1:1000; Cell Signaling Technology) at $4^{\circ} \mathrm{C}$ overnight, followed by incubation with horseradish peroxidase-conjugated secondary antibodies (Santa Cruz Biotechnology, Santa Cruz, CA, USA) for $1 \mathrm{~h}$. Protein bands were visualized using SuperSignal enhanced chemiluminescence kit (Pierce, Rockford, IL, USA).

\section{RNA immunoprecipitation assay}

RNA immunoprecipitation (RIP) assay was performed with anti-Agol (Abcam, Cambridge, MA, USA) or IgG (Sigma-Aldrich). SMMC7721 cells at $48 \mathrm{~h}$ after transfection with miR-506 or miR-NC were lysed in complete RIP lysis buffer, and then 100 $\mu \mathrm{L}$ whole-cell lysate of SMMC7721 cells was incubated with RIP buffer containing magnetic beads conjugated with human anti-Agol or negative control IgG. Samples were incubated with Proteinase K with shaking to digest the protein, and RNA coimmunoprecipitated with anti-Agol or IgG antibodies was isolated using TRIzol (Invitrogen) and then subjected to qRTPCR analysis.

\section{Statistical analysis}

All experimental results are shown as mean $\pm \mathrm{SD}$, and all statistical analyses were performed using SPSS 19.0 (IBM Corp., Armonk, NY, USA). Comparison between two or more groups was performed using Student's t-test and one-way ANOVA. A twotailed $p<0.05$ was considered statistically significant.

\section{RESULTS}

miR-506 expression was positively correlated with NK cell cytotoxicity against HCC cells

To characterize the function of miR-506 in NK cells, we determined the expression of miR-506 in primary NK cells isolated from HCC patients and healthy controls. As shown in Fig. 1A, miR-506 expression was significantly downregulated in primary NK cells from 15 HCC patients as compared with that from 15 healthy controls. In addition, primary NK cells from HCC patients showed remarkably reduced cytotoxicity against SMMC7721 or HepG2 cells, with respect to primary NK cells from healthy controls (Fig. 1B and 1C). Interestingly, we found that miR-506 expression was positively correlated with NK cell cytotoxicity against SMMC7721 or HepG2 cells (Fig. 1D and E).

\section{miR-506 overexpression enhanced NK cell cytotoxicity against HCC cells}

To further validate the role of miR-506 in NK cells, gain-of-function and loss-of-function approaches were performed in NK92 and primary NK cells by transfecting with miR-506, antimiR-506, or matched controls. qRT-PCR analyses demonstrated that miR-506 expression was dramatically elevated in miR506-transfected NK-92 and primary NK cells, but significantly declined in anti-miR-506-treated NK-92 and primary NK cells (Fig. 2A and B). Moreover, CFSE/7AAD cytotoxicity assay showed that cytotoxicity of miR-506-mimicked NK-92 cells to SMMC7721 cells was evidently higher than that of miR-NCtransfected cells (Fig. 2C). On the contrary, anti-miR-506-introduced NK-92 cells exhibited decreased cytotoxicity to HepG2 cells at different E:T ratios relative to anti-miR-NC group (Fig. 2D). Consistently with the results from CFSE/7AAD cytotoxicity assay, LDH assay demonstrated that enhanced expression of miR-506 by miR-506 mimic drastically increased primary NK cell cytotoxicity at E:T of 5:1, while knockdown of miR-506 by anti-miR-506 strikingly inhibited the cytotoxicity of primary NK cells from HCC patients against SMMC7721 or HepG2 cells (Fig. 2E and F). Collectively, these results demonstrated that miR-506 overexpression enhanced NK cell cytotoxicity against HCC cells.

\section{STAT3 was a target of miR-506}

To further investigate the underlying mechanism of miR-506 in regulating NK cell cytotoxicity against HCC cells, bioinformatics analysis was performed to predict the potential targets 

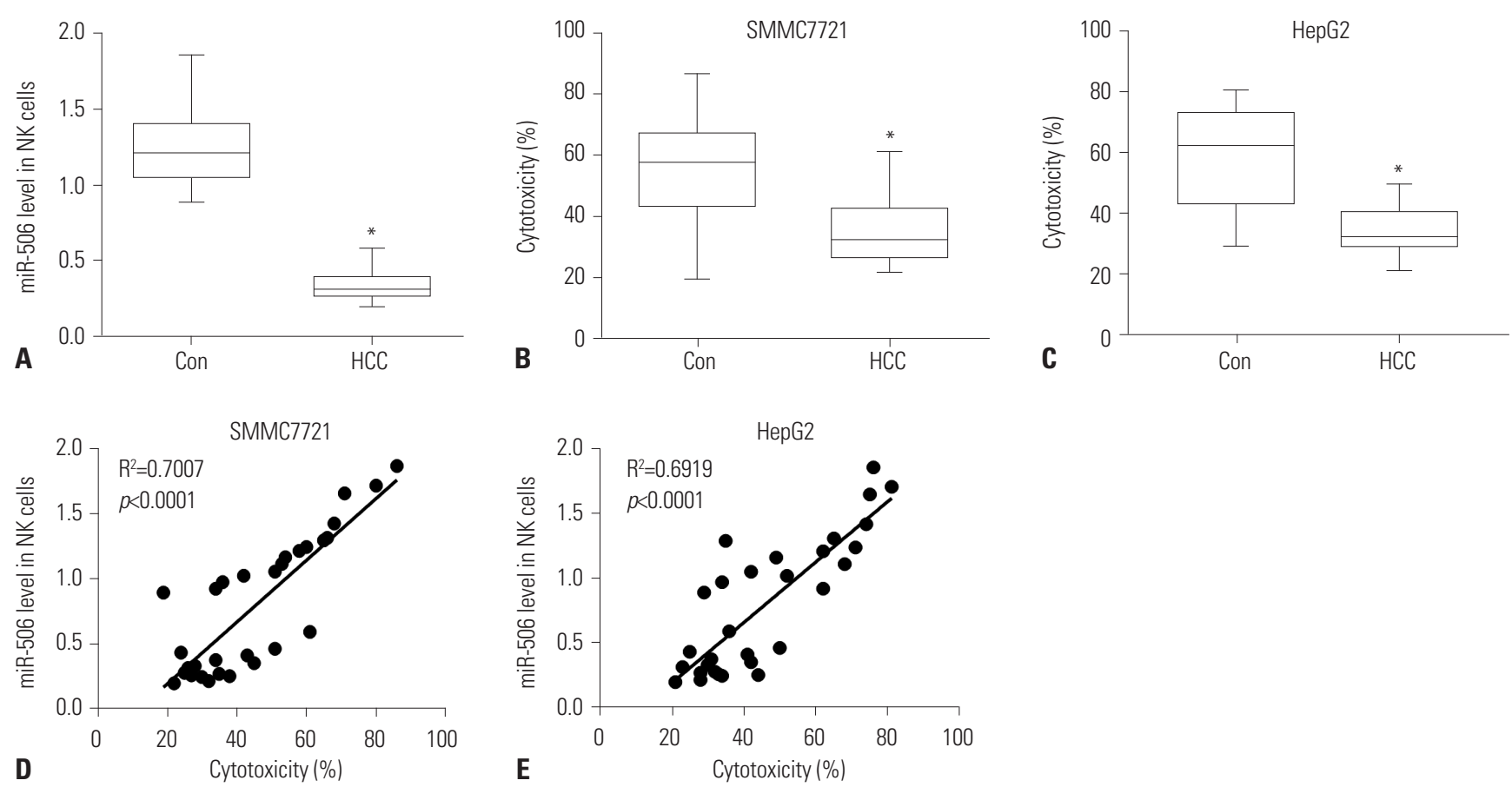

Fig. 1. miR-506 expression was positively correlated with NK cell cytotoxicity against SMMC7721 cells. (A) Quantitative real-time PCR analysis of miR506 in primary NK cells isolated from 15 HCC patients and 15 healthy controls. (B and C) Primary NK cell cytotoxicity against SMMC7721 (B) or HepG2 (C) cells was detected by lactate dehydrogenase cytotoxicity assay. (D and E) Correlation between miR-506 expression and NK cell cytotoxicity against SMMC7721 (D) or HepG2 (E) cells. * $p<0.05$. NK, natural killer; HCC, hepatocellular carcinoma.

of miR-506. The results demonstrated that STAT3 was predicted as a target of miR-506 based on putative target sequence at position 1303-1309 of STAT3 3'UTR, as shown in Fig. 3A. To validate whether STAT3 was a direct target of miR-506, luciferase reporter plasmids containing wild-type or mutated STAT3 3'UTR were constructed and luciferase reporter assay was performed. As displayed in Fig. 3B, overexpression of miR-506 led to a remarkable reduction of luciferase activity of STAT3WT in 293T cells, but did not affect luciferase activity of STAT3MUT. To further explore whether miR-506 and STAT3 mRNA interact and form miRNA ribonucleoprotein (miRNP) complexes, RIP assay was conducted and the results implicated that STAT3 could be specifically recruited to the miRNP complexes isolated using Agol antibody following forced expression of miR-506 (Fig. 3C). qRT-PCR analysis demonstrated that STAT3 mRNA expression was aberrantly higher in primary NK cells from 15 HCC patients compared to that from 15 healthy controls (Fig. 3D), and negatively correlated with miR506 expression in HCC patients (Fig. 3E). Moreover, we found that miR-506 mimic-transfected primary NK cells exhibited a significant decrease of STAT3 level, while anti-miR-506-treated primary NK cells displayed an evident increase of STAT3 level (Fig. 3F). Therefore, we concluded that miR-506 could suppress STAT3 expression by directly targeting 3'UTR of STAT3.

miR-506 overexpression enhanced NK cell cytotoxicity against HCC cells by targeting STAT3

We found that STAT3 mRNA expression was negatively corre- lated with NK cell cytotoxicity against SMMC7721 cells (Fig. 4A). To demonstrate the effects of miR-506-STAT3 axis on NK cell cytotoxicity, NK-92 and primary NK cells were transfected with miR-NC, miR-506, miR-506 + pcDNA, miR-506 + STAT3, anti-miR-NC, anti-miR-506, anti-miR-506 + si-NC, or antimiR-506 + si-STAT3. Unsurprisingly, STAT3 mRNA level was inhibited in NK-92 or primary NK cells transfected with miR506 , whereas introduction of STAT3 overexpression supported the abundance (Fig. 4B-E). Subsequently, CFSE/7AAD cytotoxicity assay showed that ectopic expression of miR-506 strikingly promoted cytotoxicity of NK-92 cells against SMMC7721 cells, while cotransfection with miR-506 and STAT3 dismissed this effect (Fig. 4F). In contrast, STAT3 knockdown significantly abolished anti-miR-506-mediated suppression of NK cell cytotoxicity against HepG2 cells at different E:T ratios (Fig. 4G). LDH assay also revealed that increased expression of miR-506 distinctly accelerated the cytotoxic activity of primary NK cells from HCC patients against SMMC7721 cells, which was obviously counteracted after overexpressing STAT3 (Fig. 4H). On the contrary, anti-miR-506-mediated inhibition of the cytotoxic activity of primary NK cells from HCC patients against HepG2 cells was dramatically abolished following STAT3 knockdown (Fig. 4I). Taken together, these results demonstrated that overexpressing STAT3 greatly reversed miR-506-mediated promotion of NK cell cytotoxicity against HCC cells. 

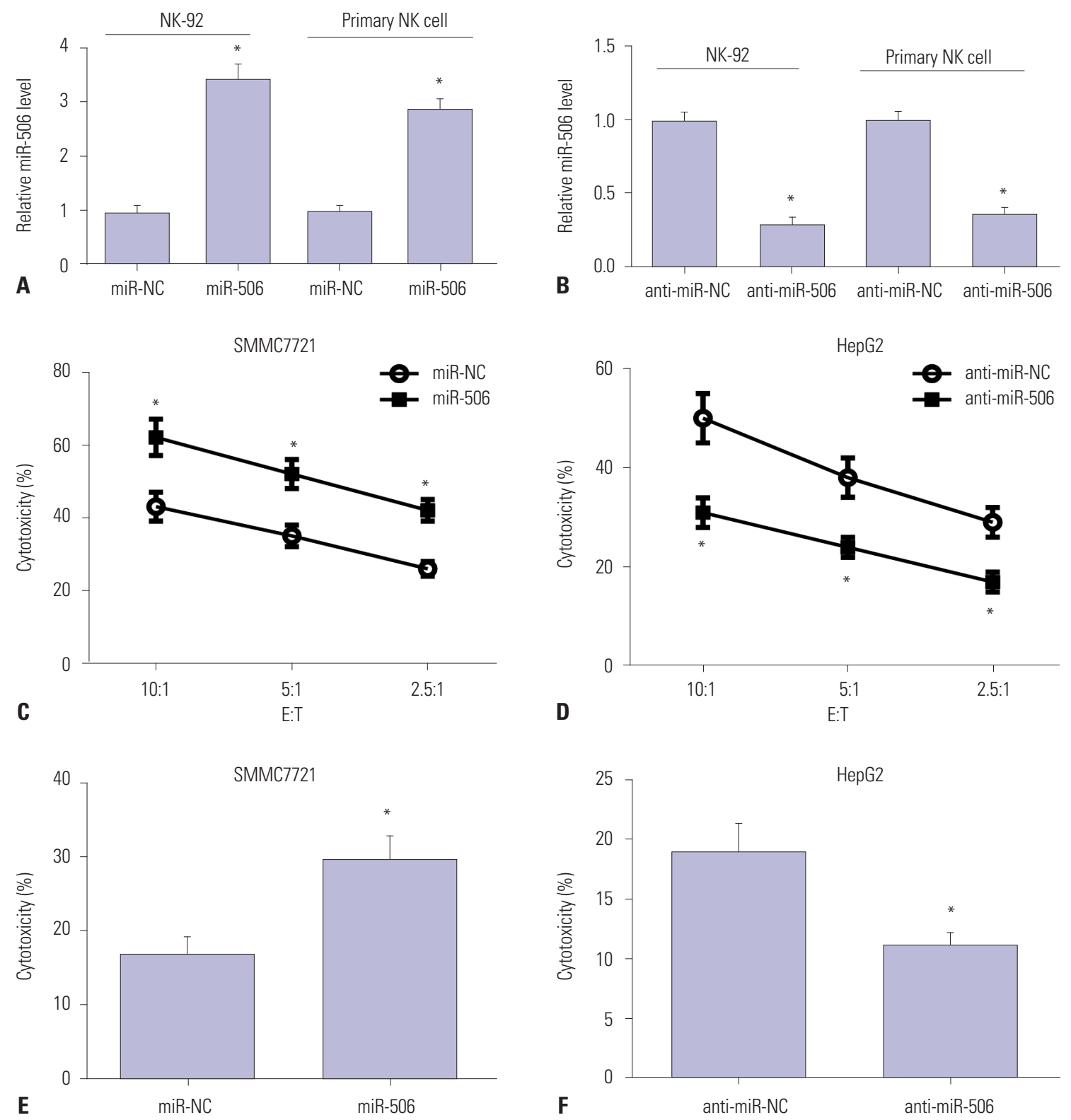

Fig. 2. miR-506 overexpression enhanced NK cell cytotoxicity against HCC cells. (A and B) NK-92 and primary NK cells were transfected with miR-506, anti-miR-506, or matched controls, and miR-506 expression was then examined by quantitative real-time PCR. (C and D) NK-92 cell-mediated cytotoxicity against SMMC7721 (C) or HepG2 (D) cells was assessed via flow cytometry using CFSE/7AAD cytotoxicity assay. (E and F) Primary NK cell cytotoxicity against SMMC7721 (E) or HepG2 (F) cells was determined using lactate dehydrogenase assay. * $p<0.05$. NK, natural killer; HCC, hepatocellular carcinoma; E:T, effector-to-target ratios.

\section{DISCUSSION}

In the immune system, miRNAs with known function play an important role in the development and activation of immune cells, as well as immune responses..$^{13}$ In recent years, increasing studies have focused on the emerging roles of miRNA in regulation of activation and cytotoxic function of NK cells in tumor cells. For example, enforced expression of miR-889 attenuated the susceptibility of HCC cells to NK cell-mediated lysis by targeting major histocompatibility complex class I chain-related gene $\mathrm{B}$ (MICB), which participated in NK cell-mediated antitumor immune response. ${ }^{14}$ In addition, increased levels of
miR-20a in ovarian cancer cells indirectly suppressed NK cell cytotoxicity by downregulating MICA/B expression. ${ }^{15}$ Moreover, upregulated miR-146a expression reduced NK cell-mediated cytotoxicity in chronic hepatitis B-infected patients and HCC patients by targeting STAT $1 .{ }^{16}$ In the present study, we addressed the roles of miR-506 in regulating NK cells cytotoxicity against HCC cells and the underlying mechanism.

The role of miR-506 in different types of cancer is complicated, even contradictory. miR-506 has been reported to be downregulated in addition to acting as a tumor suppressor in osteosarcoma, ${ }^{17}$ esophageal cancer, ${ }^{18}$ breast cancer, ${ }^{19}$ and HCC. ${ }^{20}$ On the contrary, it was convincingly demonstrated that miR- 
506 played an oncogenic role in melanomas ${ }^{21}$ and colon cancer. ${ }^{22}$ However, the roles of miR-506 in regulating NK cells cytotoxicity against HCC cells were still unknown. In our study, we demonstrated that miR-506 expression was significantly downregulated in primary NK cells from HCC patients compared to that from healthy controls. In addition, we found that
Position 1303-1309 of STAT3 3' UTR

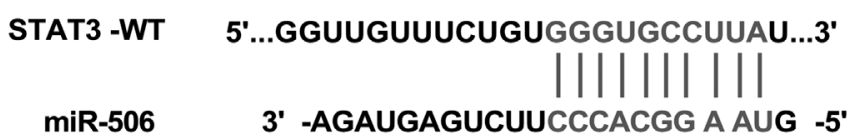

STAT3-MUT

A miR-506

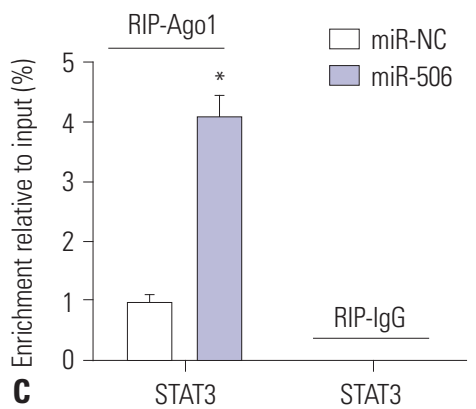

5'...GGUUGUUUCUGUUUAGAUUGGCU...3'

3'- AGAUGAGUCUUCCCACGgAUG -5'
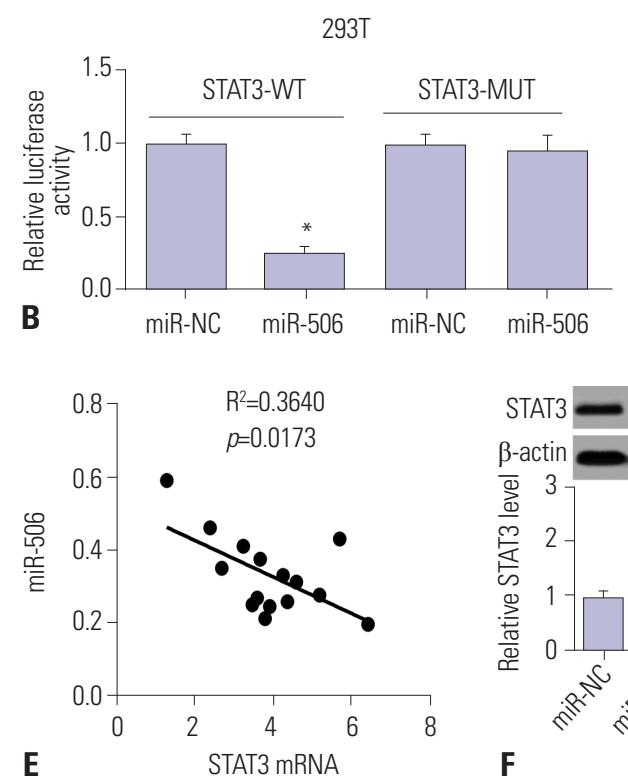

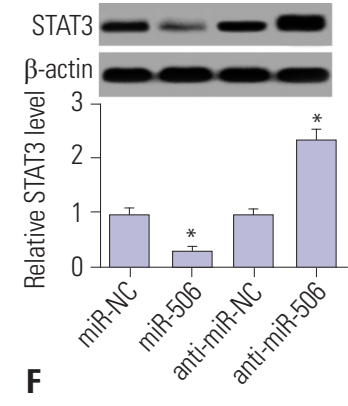

Fig. 3. Interaction between miR-506 and STAT3. (A) Predicted potential binding sites of miR-506 to 3'UTR of STAT3. (B) Luciferase reporter assay was performed to detect luciferase activity in 293T cells after cotransfection with miR-506 or miR-NC and luciferase reporter vectors containing wild-type or mutated STAT3 3'UTR. (C) Quantitation of recruited mRNAs of STAT3 to miRNP complex immunoprecipitated with Ago1 by RIP analysis. (D) mRNA expression of STAT3 in primary NK cells from $15 \mathrm{HCC}$ patients and healthy controls by quantitative real-time PCR. (E) Spearman correlation analysis between miR-506 and STAT3 mRNA expressions in HCC patients. (F) STAT3 level was detected in primary NK cells transfected with miR-506, anti-miR-506, or matched controls using western blot. ${ }^{*} p<0.05$. NK, natural killer; HCC, hepatocellular carcinoma; E:T, effector-to-target ratios; STAT3, signal transducer and activator of transcription 3; miRNP, miRNA ribonucleoprotein; RIP, RNA immunoprecipitation; 3'-UTR, 3'-untranslated regions.
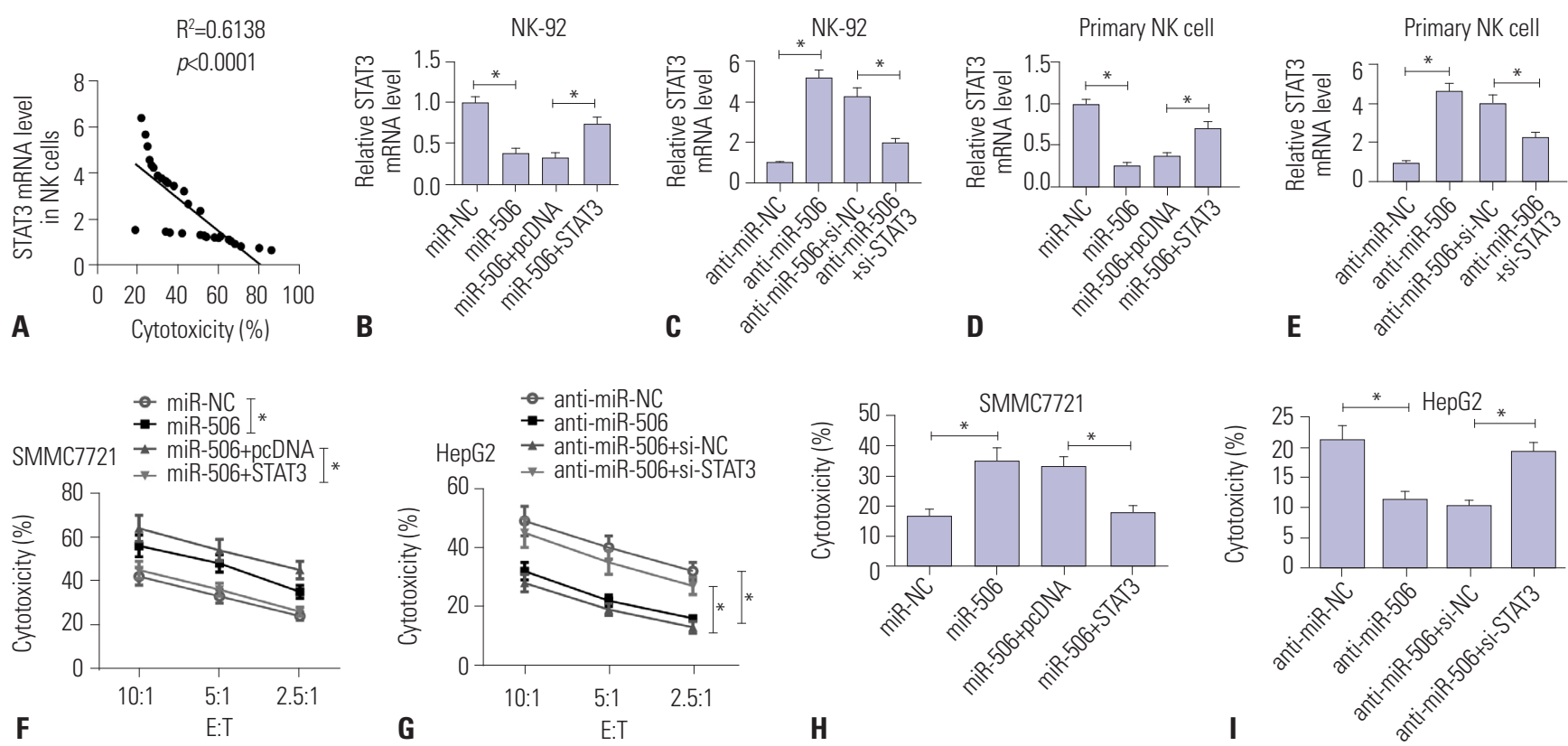

Fig. 4. miR-506 overexpression enhanced NK cell cytotoxicity against HCC cells by targeting STAT3. (A) Correlation between STAT3 mRNA expression and NK cell cytotoxicity. (B and C) NK-92 cells were transfected with miR-NC, miR-506, miR-506 + pcDNA, miR-506 + STAT3, anti-miR-NC, antimiR-506, anti-miR-506 + si-NC, or anti-miR-506 + si-STAT3, and mRNA expression of STAT3 was examined. (D and E) Primary NK cells were treated with miR-NC, miR-506, miR-506 + pcDNA, miR-506 + STAT3, anti-miR-NC, anti-miR-506, anti-miR-506 + si-NC, or anti-miR-506 + si-STAT3, and then STAT3 mRNA abundance was detected. Cytotoxicity of NK-92 cells against SMMC7721 (F) or HepG2 cells (G) was assessed via flow cytometry using CFSE/7AAD cytotoxicity assay. Cytotoxicity against SMMC7721 (H) or HepG2 cells (I) was then determined using lactate dehydrogenase assay. ${ }^{*} p<0.05$. NK, natural killer; HCC, hepatocellular carcinoma; E:T, effector-to-target ratios; STAT3, signal transducer and activator of transcription 3. 
primary NK cells from HCC patients showed remarkably reduced cytotoxicity against SMMC7721 or HepG2 cells with respect to primary NK cells from healthy controls, which was consistent with previous studies. ${ }^{4,5}$ Moreover, miR-506 was positively correlated with NK cell cytotoxicity against SMMC7721 or HepG2 cells, suggesting that miR-506 might be associated with NK cell cytotoxicity in HCC. As expect, functional analysis demonstrated that miR-506 overexpression enhanced NK cell cytotoxicity against HCC cells, while miR-506 inhibitor showed the reverse effect.

To further explore the molecular mechanism by which miR506 enhanced NK cell cytotoxicity against HCC cells, we predicted the potential targets of miR-506 using bioinformatics analysis. STAT3 was identified as a direct target of miR-506 by luciferase reporter assay, and RIP and miR-506 suppressed STAT3 expression. Constitutive activation of STAT3 is observed in diverse human tumors, and STAT3 has been reported to mediate the crosstalk between tumor and immune cells. ${ }^{23}$ Moreover, aberrant activation of STAT3 contributes to tumor immune evasion via inhibiting antitumor immunity. ${ }^{24}$ It has been proposed that STAT3 regulated NK cytolytic activity, as well as reciprocal regulatory interaction between NK cells and other components of the immune system. ${ }^{25}$ Blockage of STAT3 in HCC cells augmented the cytotoxicity of NK cells by reversing HCC-induced immune suppression. ${ }^{26}$ Our study provided the evidence that STAT3 mRNA expression was significantly upregulated in primary NK cells from HCC patients and negatively correlated with NK cell cytotoxicity against SMMC7721 or HepG2 cells. Rescue experiments demonstrated that overexpressing STAT3 greatly reversed miR-506-mediated promotion of NK cell cytotoxicity against HCC cells, suggesting that miR506 overexpression enhanced NK cell cytotoxicity against HCC cells by targeting STAT3.

In summary, we firstly demonstrated that miR-506 was downregulated while STAT3 was upregulated in primary NK cells from HCC patients. Moreover, we found that miR-506 overexpression enhanced NK cell cytotoxicity against HCC cells by targeting STAT3. Therefore, targeting miR-506 may be a promising new therapeutic strategy for enhancing NK cell-mediated immunotherapy to treat HCC.

\section{ACKNOWLEDGEMENTS}

This study was approved by Guangxi Health Department Selffinancing Project (Grant No. Z2015541).

\section{ORCID iDs}

Zhixiong Su Xinping Ye Liming Shang https://orcid.org/0000-0002-3497-2073 https://orcid.org/0000-0002-9959-5272 https://orcid.org/0000-0002-0191-3464

\section{REFERENCES}

1. Torre LA, Bray F, Siegel RL, Ferlay J, Lortet-Tieulent J, Jemal A. Global cancer statistics, 2012. CA Cancer J Clin 2015;65:87-108.

2. Waldhauer I, Steinle A. NK cells and cancer immunosurveillance. Oncogene 2008;27:5932-43.

3. Ishiyama $\mathrm{K}$, Ohdan $\mathrm{H}$, Ohira M, Mitsuta $\mathrm{H}$, Arihiro $\mathrm{K}$, Asahara T. Difference in cytotoxicity against hepatocellular carcinoma between liver and periphery natural killer cells in humans. Hepatology 2006;43:362-72.

4. Subleski JJ, Hall VL, Back TC, Ortaldo JR, Wiltrout RH. Enhanced antitumor response by divergent modulation of natural killer and natural killer T cells in the liver. Cancer Res 2006;66:11005-12.

5. Zhang QF, Yin WW, Xia Y, Yi YY, He QF, Wang X, et al. Liver-infiltrating CD11b-CD27- NK subsets account for NK-cell dysfunction in patients with hepatocellular carcinoma and are associated with tumor progression. Cell Mol Immunol 2017;14:819-29.

6. Hoechst B, Voigtlaender T, Ormandy L, Gamrekelashvili J, Zhao F, Wedemeyer $\mathrm{H}$, et al. Myeloid derived suppressor cells inhibit natural killer cells in patients with hepatocellular carcinoma via the NKp30 receptor. Hepatology 2009;50:799-807.

7. Gu S, Jin L, Zhang F, Sarnow P, Kay MA. Biological basis for restriction of microRNA targets to the 3' untranslated region in mammalian mRNAs. Nat Struct Mol Biol 2009;16:144-50.

8. Svoronos AA, Engelman DM, Slack FJ. OncomiR or tumor suppressor? The duplicity of microRNAs in cancer. Cancer Res 2016;76: 3666-70.

9. O'Connell RM, Baltimore D. MicroRNAs and hematopoietic cell development. Curr Top Dev Biol 2012;99:145-74.

10. Squadrito ML, Etzrodt M, De Palma M, Pittet MJ. MicroRNA-mediated control of macrophages and its implications for cancer. Trends Immunol 2013;34:350-9.

11. Bentwich I, Avniel A, Karov Y, Aharonov R, Gilad S, Barad O, et al. Identification of hundreds of conserved and nonconserved human microRNAs. Nat Genet 2005;37:766-70.

12. Li J, Ju J, Ni B, Wang $\mathrm{H}$. The emerging role of miR-506 in cancer. Oncotarget 2016;7:62778-88.

13. Kumar Kingsley SM, Vishnu Bhat B. Role of MicroRNAs in the development and function of innate immune cells. Int Rev Immunol 2017;36:154-75.

14. Xie H, Zhang Q, Zhou H, Zhou J, Zhang J, Jiang Y, et al. microRNA-889 is downregulated by histone deacetylase inhibitors and confers resistance to natural killer cytotoxicity in hepatocellular carcinoma cells. Cytotechnology 2018;70:513-21.

15. Xie J, Liu M, Li Y, Nie Y, Mi Q, Zhao S. Ovarian tumor-associated microRNA-20a decreases natural killer cell cytotoxicity by downregulating MICA/B expression. Cell Mol Immunol 2014;11:495502.

16. Xu D, Han Q, Hou Z, Zhang C, Zhang J. miR-146a negatively regulates NK cell functions via STAT1 signaling. Cell Mol Immunol 2017; 14:712-20.

17. Yu Z, Zhang Y, Gao N, Wang X. Overexpression of miR-506 inhibits growth of osteosarcoma through Snail2. Am J Transl Res 2015;7: 2716-23.

18. Yao WJ, Wang YL, Lu JG, Guo L, Qi B, Chen ZJ. MicroRNA-506 inhibits esophageal cancer cell proliferation via targeting CREB1. Int J Clin Exp Pathol 2015;8:10868-74.

19. Arora H, Qureshi R, Park WY. miR-506 regulates epithelial mesenchymal transition in breast cancer cell lines. PLoS One 2013;8: e64273.

20. Dai W, Huang HL, Hu M, Wang SJ, He HJ, Chen NP, et al. microRNA-506 regulates proliferation, migration and invasion in hepato- 
cellular carcinoma by targeting F-spondin 1 (SPON1). Am J Cancer Res 2015;5:2697-707.

21. Streicher KL, Zhu W, Lehmann KP, Georgantas RW, Morehouse CA, Brohawn P, et al. A novel oncogenic role for the miRNA-506514 cluster in initiating melanocyte transformation and promoting melanoma growth. Oncogene 2012;31:1558-70.

22. Tong JL, Zhang CP, Nie F, Xu XT, Zhu MM, Xiao SD, et al. MicroRNA 506 regulates expression of PPAR alpha in hydroxycamptothecin-resistant human colon cancer cells. FEBS Lett 2011;585:3560-8.

23. Yu H, Kortylewski M, Pardoll D. Crosstalk between cancer and immune cells: role of STAT3 in the tumour microenvironment. Nat
Rev Immunol 2007;7:41-51.

24. Wang T, Niu G, Kortylewski M, Burdelya L, Shain K, Zhang S, et al. Regulation of the innate and adaptive immune responses by Stat3 signaling in tumor cells. Nat Med 2004;10:48-54.

25. Cacalano NA. Regulation of natural killer cell function by STAT3. Front Immunol 2016;7:128.

26. Sun X, Sui Q, Zhang C, Tian Z, Zhang J. Targeting blockage of STAT3 in hepatocellular carcinoma cells augments NK cell functions via reverse hepatocellular carcinoma-induced immune suppression. Mol Cancer Ther 2013;12:2885-96. 